\title{
The pulmonary angiographic appearance of pleurisy associated with subdiaphragmatic inflammation
}

\author{
S W BANHAM, A D HOWIE, R D STEVENSON, AND F MORAN \\ From the Centre for Respiratory Investigation, Royal Infirmary, Glasgow, UK
}

ABSTRACT In two patients who had recently undergone major abdominal operations and were later suspected of having pulmonary emboli, pulmonary angiography showed no evidence of embolism, but in both cases one of the hemidiaphragms was clearly outlined by contrast material. It is suggested that subdiaphragmatic inflammation was responsible for this unusual appearance.

\section{Case 1}

Four weeks after cholecystectomy a 31-year-old woman was admitted to hospital with abdominal pain and vomiting. A cholangiogram showed stones remaining in the common bile duct. At laparotomy the calculi were removed and a choledochojejunostomy constructed. Two days later she developed fever associated with left hypochondrial pain. Left pleuritic pain followed, and a pleural friction rub was heard. The chest radiograph showed consolidation/collapse of the left lower lobe. Pulmonary embolism was suspected, but on angiography none of the characteristic features was shown, although changes were noted over the left hemidiaphragm (fig 1). Diaphragmatic screening established the presence

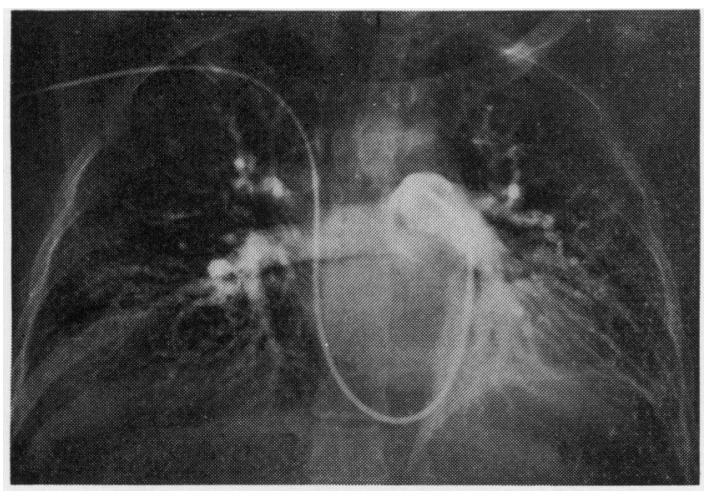

Fig 1 Pulmonary angiogram two seconds after contrast injection in a 31-year-old woman with a left subphrenic collection. Left hemidiaphragm is outlined by contrast. Despite density of contrast at left base this cleared normally in subsequent films. of a subphrenic collection communicating with the wound site. Thereafter, with appropriate antibiotic treatment, the patient made a rapid and uneventful recovery.

\section{Case 2}

While being investigated for chronic relapsing pancreatitis, a 49-year-old man developed acute abdominal pain associated with a considerably raised serum amylase concentration (3100 U/1). The pain settled on conservative treatment, but in view of the long history of severe symptoms he underwent an operation involving splenectomy, partial pancreatectomy, and a pancreaticojejunostomy. There were no postoperative complications until the seventh day. Then after removal of a drainage tube from the left subphrenic region he became distressed by left hypochondrial pain and breathlessness. Examination showed considerable tenderness around the draining site and reduced breath sounds at the left base. A chest radiograph 24 hours later showed elevation of the left hemidiaphragm with a small effusion. A pulmonary angiogram showed no evidence of embolism, but there were abnormalities at the left base (fig 2). The pain gradually settled with analgesics, and the patient made a good recovery.

\section{Discussion}

These patients showed a remarkably similar pulmonary angiographic appearance, the striking feature being the delineation of the diaphragm with an arc of contrast material during the arterial phase. This was unlikely to have been caused by extravasation, since serial films did not show the 


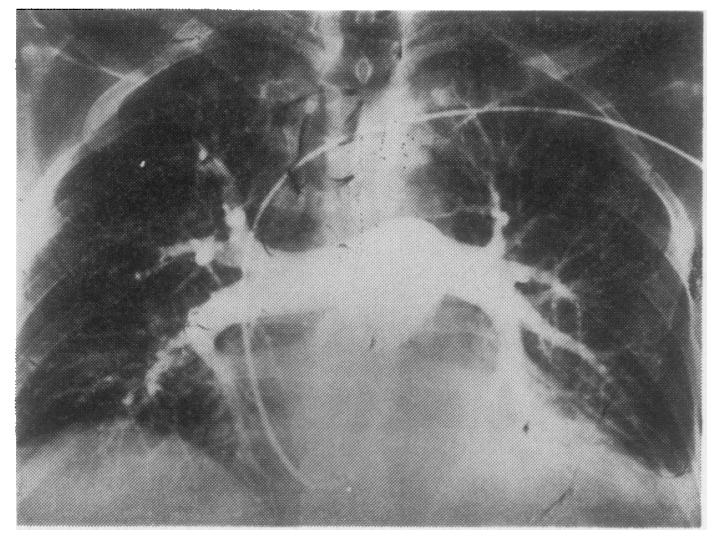

Fig 2 Pulmonary angiogram two seconds after contrast injection in a 47-year-old man with left chest pain. Left hemidiaphragm delineated by contrast, but no vessel occlusion suggestive of embolism. Contrast cleared normally from both lower lobes.

typical delayed clearance of contrast medium. Furthermore, there was no angiographic evidence of pulmonary embolism (Sasahara et al, 1964; Stein et al, 1967). In our experience this radiological appearance is rare. No similar cases were found on review of over 1000 pulmonary angiograms performed in the Centre for Respiratory Investigation in Glasgow Royal Infirmary since 1968. There were several patients with an unexplained pneumonia or atelectasis who underwent angiography to exclude pulmonary embolism, but none of them showed this picture. Furthermore, the various angiographic abnormalities reported in other respiratory diseases (Wiener et al, 1966; Ferris et al, 1967) do not include this appearance.

In both cases there was evidence suggesting that the pulmonary changes were secondary to subdiaphragmatic inflammation. The first patient had a subphrenic infection, and in the second patient the changes could be attributed to the local inflammatory response after major pancreatic surgery. We postulate, therefore, that pleurisy secondary to subdiaphragmatic inflammation, associated with lung hyperaemia, produced the appearances described here.

\section{References}

Ferris, E J, Stanzler, R M, Rourke, J A, Blumenthal, $\mathrm{J}$, and Messer, J V (1967). Pulmonary angiography in pulmonary embolic disease. American Journal of Roentgenology, 100, 355-363.

Sasahara, A A, Stein, M, Simon, N, and Littmann, D (1964). Pulmonary angiography in the diagnosis of thrombo-embolic disease. New England Journal of Medicine, 270, 1075-1081.

Stein, P D, O'Connor, J F, Dalen, J El, Pur-Shahriari, A A, Hoppin, F F, Hammond, D T, Haynes, F W, Fleischner, F G, and Dexter, L (1967). The angiographic diagnosis of acute pulmonary embolism. Evaluation of criteria. American Heart Journal, 73, 730-741.

Wiener, S N, Edelstein, J, and Charms, B L (1966). Observations on pulmonary embolism and the pulmonary angiogram. American Journal of Roentgenology, 98, 859-873.

Requests for reprints to: Dr S W Banham, Centre for Respiratory Investigation, Royal Infirmary, Glasgow G4 OSF. 